\title{
A trimodal resonator for three mutually perpendicular magnetic fields
}

\author{
T. H. Stievater, ${ }^{\text {a) }}$ M. J. Lim, P. Colosimo, R. A. Michniak, P. H. Bucksbaum, \\ and R. S. Conti \\ Department of Physics, University of Michigan, Ann Arbor, Michigan 48109
}

(Received 17 July 1998; accepted for publication 24 November 1998)

\begin{abstract}
We describe a trimodal resonator for the simultaneous delivery of three perpendicular magnetic fields. The resonator consists of a shielded loop single-mode radio frequency (rf) resonator placed inside of a bimodal waveguide microwave resonator. The microwave modes are nondegenerate, tunable over a range of $100 \mathrm{MHz}$, and have typical Q factors of 150 and 200. The rf mode is tunable over a range of $125 \mathrm{MHz}$, and has a typical Q of 45 . Control of the relative phase between the three fields is demonstrated. The resonator will be used to drive three magnetic dipole transitions coherently between Zeeman states in the ground state of ${ }^{87} \mathrm{Rb}$. (C) 1999 American Institute of Physics. [S0034-6748(99)00403-7]
\end{abstract}

\section{INTRODUCTION}

We have successfully designed and constructed a triply resonant device capable of simultaneously delivering three perpendicular nondegenerate magnetic field standing waves with a well-characterized mutual phase relationship. This trimodal resonator is constructed by placing a shielded loop coaxial resonator inside a bimodal waveguide resonant cavity. The shielded loop mode is typically $\mathrm{rf}(<1 \mathrm{GHz})$, whereas the bimodal waveguide modes are typically microwave $(>1 \mathrm{GHz})$. Each mode is independently tunable over a range that depends upon the details of the tuning method. We have designed the trimodal resonator such that the sum of the lower two frequencies is equal to the third frequency.

The three modes of the resonator will be used to drive magnetic dipole transitions between three sublevels of the $5 \mathrm{~S}_{1 / 2}$ ground state of atomic rubidium, permitting observation of phase-dependent population dynamics. In particular, control of the individual phase of each of the three magnetic fields allows for detection of interference effects between competing channels in the excitation loop. ${ }^{1}$ Such population dynamics can reveal time-reversal symmetry breaking effects that may be present in the atomic system. ${ }^{2}$

\section{MOTIVATION}

The ability to drive three transitions between three states of an atom simultaneously, such that the highest of the three transition frequencies is equal to the sum of the lower two, allows one to study coherence in the atomic system. To the authors' knowledge, no experimental work has been done pertaining to triply driven three-state systems. These types of closed excitation loops exhibit population dynamics that differ in one important respect from both singly driven two level systems and doubly driven three level systems: Triply driven coherent three-state systems have the potential for population evolution that is directional in time. The three continuous wave $(\mathrm{cw})$ driving fields in our system can be

${ }^{a)}$ Electronic mail: tstievat@umich.edu. written as $\mathbf{B}_{i} \sin \left(\omega_{i} t+\varphi_{i}\right), i=1,2,3$. Since $\omega_{2}+\omega_{3}=\omega_{1}$, (see Fig. 1) there are effectively two fields at $\omega_{1}$ applied to the population of rubidium atoms: $\mathbf{B}_{1}$ and $\mathbf{B}_{2}+\mathbf{B}_{3}$. The phase difference between these two fields is simply

$$
\Phi=\varphi_{2}+\varphi_{3}-\varphi_{1}
$$

and is the net phase around the three state loop. It is the only phase that has physical significance for the population dynamics and depends on the relative phases of the applied fields, not on any external phase reference. The choice of a particular value of $\Phi$ can make the population preferentially cycle from $A \rightarrow B \rightarrow C \rightarrow A$ etc., where $A, B$, and $C$ are the three states of the system. Conversely, a shift in the overall phase $\Phi$ of the magnetic fields can force the system to cycle preferentially from $A \rightarrow C \rightarrow B \rightarrow A$ etc. Though such directional coherent population dynamics are interesting in their own right, ${ }^{1}$ our experiment will reverse this argument. Choosing $\Phi$ such that the applied fields are time-reversal invariant should result in adirectional population dynamics. Thus, any observation of temporal directionality would imply a violation of time-reversal symmetry in the atomic system. $^{2}$

Our application requires driving the three magnetic dipole transitions that couple three Zeeman substates of the $5 \mathrm{~S}_{1 / 2}$ ground state of atomic rubidium. A rubidium/buffer gas vapor cell will be placed in the center of the resonator, with an interaction region defined by the overlap of one initial state preparation laser and two final state detection lasers (a few $\mathrm{mm}^{3}$ ), as discussed below. The application of three $\mathrm{cw}$ perpendicular magnetic fields will be used to couple the substates, since a field parallel to the direction of the dc magnetic field (the $z$ direction) can couple $\Delta m=0$ transitions, and the other two fields in the $x$ and $y$ directions can couple $\Delta m= \pm 1$ transitions, as shown in Fig. 1. Our three magnetic fields need only be mutually perpendicular within the interaction region at the center of the resonator. In addition, the $\mathrm{Q}$ factors for the three magnetic fields need to be high enough to generate field magnitudes sufficient to couple the states, but not so high that off-resonant phase shifts become problematic. Q's of about 100 turn out to be in the right range. 


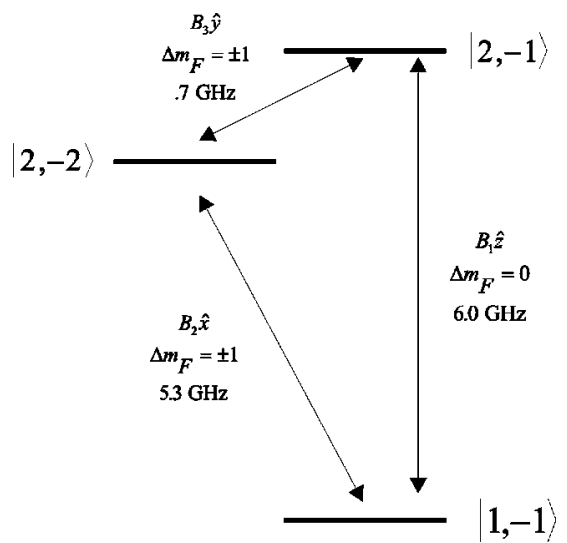

FIG. 1. Three Zeeman substates (labeled in a $\left|F, m_{F}\right\rangle$ basis) of the ${ }^{87} \mathrm{Rb} 5 \mathrm{~S}_{1 / 2}$ ground state, coupled by magnetic dipole transitions at a dc bias field of 806 Gauss. The frequencies and polarizations for each magnetic field are shown.

The experiment will be carried out at a static magnetic field of 806 Gauss in ${ }^{87} \mathrm{Rb}$ at transition frequencies of $727 \mathrm{MHz}$, $5.304 \mathrm{GHz}$, and $6.031 \mathrm{GHz}$ for the three states shown in Fig. 1.

The properties required of the trimodal resonator include: independent tunability of each frequency to achieve specific resonant frequencies, compensating for detuning effects inherent in loading the cavity; isolation and geometric perpendicularity of each mode at the central interaction region; moderately high $\mathrm{Q}$ factors for each field (characterized by minimal losses due to resistive heating or radiation of electric fields); homogeneity of the fields at the central interaction region; and phase control.

Various techniques from nuclear magnetic resonance and electronic spin resonance suggest some possible alternative uses for the trimodal resonator. For example, electronelectron double resonance (ELDOR) requires two simultaneously driven electronic magnetic dipole transitions, usually at microwave frequencies, while electron-neutron double resonance (ENDOR) requires driving a nuclear magnetic dipole rf transition and an electronic magnetic dipole transition. The addition of a third field opens up the possibility for even greater signal resolution and sensitivity compared to double resonance techniques. ${ }^{3}$ In an electron-nuclear-nuclear triple resonance experiment, two rf magnetic fields are used for the nuclear transitions, and one microwave magnetic field is used for the electronic transition. In an electron-nuclearelectron (ENE) triple resonance experiment, two microwave and one rf field are used. ${ }^{4}$ A trimodal cavity that produces magnetic field standing waves can be used for any of these types of cw triple resonance experiments, as well as pulsed experiments that require repetition rates or rise times no faster than the buildup time of the resonant fields in the cavity. ${ }^{5}$

Recently, polarization-enhanced nuclear magnetic resonance (NMR) spectroscopy has obtained signal enhancement by polarization transfer from free electrons to nuclear spins through microwave irradiation. ${ }^{6}$ These techniques can ideally increase NMR signal intensities by the ratio of electronic to nuclear Larmor frequencies. The use of a second microwave field for a double-polarization transfer experiment could increase the sensitivity of NMR signals even further. ${ }^{7}$ Such

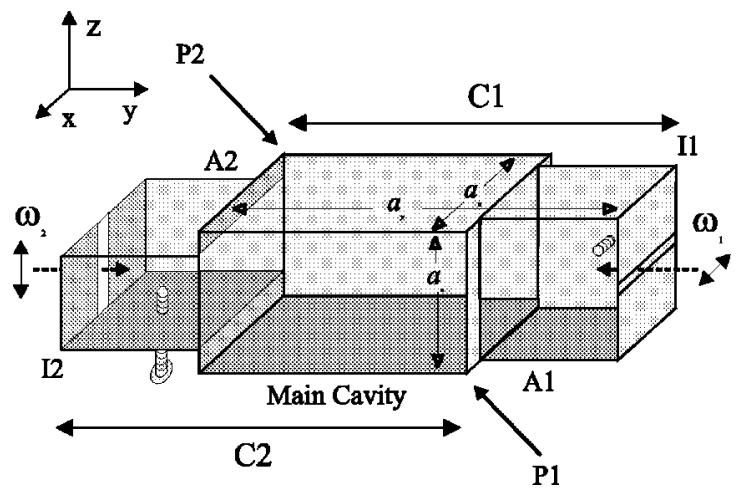

FIG. 2. The bimodal resonator used to deliver the two microwave modes. $\mathrm{A} 1(2)$ is the antechamber used with the main cavity to form effective cavity $\mathrm{C} 1(2)$. I1(2) is the input inductive iris for $B_{1(2)}$. P1(2) is a port that is transparent for $B_{1(2)}$, and reflective for $B_{2(1)}$.

experiments would require a trimodal device that is resonant at two microwave frequencies for the electronic magnetic dipole transitions and at one rf frequency for the NMR signal.

\section{DESIGN AND CONSTRUCTION OF THE TRIMODAL RESONATOR}

\section{A. The bimodal resonator}

The design of the bimodal resonator, used to deliver the two high frequency fields, is based on a design initially used for electron paramagnetic resonance (EPR) spectrometry. ${ }^{8,9}$ As shown in Fig. 2, two overlapping resonant cavities, C1 and $\mathrm{C} 2$, rotated $90^{\circ}$ with respect to one another, form the basis of the device. In a single rectangular metallic cavity, those frequencies that satisfy the boundary conditions for a transverse electric (TE) mode $\left(E_{y}=0\right.$, tangential components of $\mathbf{E}$ and normal component of $\mathbf{B}$ vanish at all cavity walls) will yield a large resonant standing wave field within the cavity. The condition for resonance is given by

$$
\omega_{0}=c \pi \sqrt{\left(\frac{n_{x}}{a_{x}}\right)^{2}+\left(\frac{n_{y}}{a_{y}}\right)^{2}+\left(\frac{n_{z}}{a_{z}}\right)^{2}},
$$

where $a_{x}, a_{y}$, and $a_{z}$ are the width, length, and height of the cavity, respectively. The parameters $n_{x}, n_{y}$, and $n_{z}$ are the mode numbers corresponding to the integer number of halfwavelengths that fit into the cavity in each of the three directions. The resonant frequency is specified by choosing the cavity dimensions to satisfy Eq. (2) for a given $\mathrm{TE}_{n_{x} n_{y} n_{z}}$ mode.

The bimodal cavity consists of a main chamber that forms part of the resonant cavity for both modes, and two antechambers (A1 and A2) that each form part of the resonant cavity for one mode only. The length of each antechamber is identical, and chosen to be half that of the main section. A $\mathrm{TE}_{031}$ mode in $\mathrm{C} 1$ and a $\mathrm{TE}_{130}$ mode in $\mathrm{C} 2$ are set up in the following way. An electromagnetic field with E-field polarized in the $x$ direction is fed into the cavity through an inductive iris, I1. P2 is reflective for this mode, since the size of the opening in the $z$ direction is below cutoff. The resonant cavity for this mode will then consist of $\mathrm{A} 1$, plus the main section. A second field at a different frequency with 
E-field polarized in the $z$ direction is fed into the cavity through the inductive iris I2. P1 is reflective for this mode. By choosing this overall mode configuration, there are two equations for two unknowns $\left(a_{x}\right.$ and $\left.a_{z}\right)$ that uniquely specify the cavity dimensions, for any given cavity length, $a_{y}$. The fact that $\mathrm{C} 1$ has a discontinuity in width, $a_{x}$, is of little consequence (as long as the dimensions of A1 do not put the mode below cutoff) since the resonant frequency does not explicitly depend on this dimension. The same is true for the height, $a_{z}$, of $\mathrm{C} 2$.

There are other advantages to this mode configuration. The electric field nodes and magnetic field antinodes for both fields occur at the center of the main section, since there are roughly three half-wavelengths in each mode's effective cavity. Here, mode one has a magnetic field $\left(B_{1}\right)$ polarized in the $z$ direction, and mode two has a magnetic field $\left(B_{2}\right)$ polarized in the $x$ direction $^{8}$ (as shown in Fig. 4). It is here that an experiment is performed in the cavity, exploiting the overlapping maxima in the magnitudes and zeros in the gradients of the magnetic fields.

The insertion of a dielectric screw (shown in Fig. 2) into one of the antechambers tunes the resonant frequency of that antechamber's mode by increasing the effective path length inside the resonant cavity. It does not affect the resonant frequency of the other mode, since only one mode occupies the volume of the antechamber. The position of each screw is chosen to overlap an E-field antinode, maximizing its effect on the resonant field and thus its tuning range. We have used both nylon and alumina as the tuning dielectrics $\left(\frac{3}{4}\right.$ in. long, 32 turns per inch), though the majority of the results presented are for nylon. Due to its high loss, nylon should not be used for applications requiring very high Q's. This tunability is crucial to set the resonance to the correct frequency after the sample is loaded into the interaction region, and to account for effects from holes that allow the field to bulge out of the cavity. Dielectric loading and field protrusion through holes both tend to increase the cavity's effective size, thereby decreasing its resonant frequency. Metallic slugs inserted into regions of magnetic field are also commonly used to tune the resonant frequency of cavities upward (via exclusion of magnetic flux). However, in our cavity the only place where a B-field antinode does not overlap a cavity wall is in the center of the cavity, where modeselective tuning is impossible. The dimensions of the cavity are consequently chosen such that the unloaded cavity resonant frequencies are higher than the desired atomic resonances. The resonance is then tuned downward through sample loading and other perturbations, followed by the insertion of the dielectric screws.

These perturbations should be minimized in order to maintain a high $\mathrm{Q}$ factor and to disturb the overlap of the central B-field antinodes as little as possible. The best designs are those for which the detuning due to perturbations in the main cavity is roughly twice the detuning due to the screw in the antechamber (for a given mode), and the detuning for one mode is comparable to that of the other mode.

The present design departs from older bimodal EPR spectrometers ${ }^{8,9}$ in the choice of dimensions, which govern the resonant frequencies in the manner described above. Our bimodal resonator is inherently nondegenerate since the dimensions $a_{x}$ and $a_{z}$ are chosen to be unequal, yielding resonant frequencies separated by nearly $1 \mathrm{GHz}$. Previous designs matched $a_{x}$ and $a_{z}$, producing modes that could differ in resonant frequency only through differential dielectric tuning, usually $100 \mathrm{MHz}$ or less. Although the performance of degenerate and nondegenerate prototypes that we have tested is quite similar, there are some noteworthy design considerations specific to the nondegenerate case pertaining to the choice of the cavity dimensions.

In the nondegenerate design, the impedance mismatch for mode one at P1 is different than that for mode two at P2. ${ }^{10}$ This can be ignored to first order, as long as the resonance detuning due to sample loading and tuning screws is equally distributed among the three half-wavelength sections of each cavity, maintaining E-field nodes at P1 an P2. However, the optimal design both minimizes and matches reflection of mode one at P1 and mode two at P2 (assuming large, comparable Q's are desired). This implies choosing $a_{x}$ and $a_{z}$ to be as close as possible, while still supporting nondegenerate resonances. Furthermore, the size of the noncritical antechamber dimension should match as closely as possible that dimension of the main chamber, while keeping that region below cutoff for the cross polarized mode.

On the other hand, there are other considerations that do not necessarily favor matching $a_{x}$ and $a_{z}$. For a given separation in resonant frequencies, the closer $a_{x}$ and $a_{z}$ are, the greater the difference in attenuation constants for the two antechambers. This is because there exists an optimal ratio $\lambda / \lambda_{\text {cutoff }}$ for which the attenuation constant for the propagating TE mode is minimized. ${ }^{11}$ As a consequence, the two modes will have different $Q$ factors. In practice, all of these considerations may be simplified by correctly predicting the magnitude of the sample detuning, thereby maintaining E-field nodes at P1 and P2. The precise reflectivity at these planes then becomes a secondary issue, allowing the noncritical antechamber dimensions to be chosen to minimize attenuation and maximize the $\mathrm{Q}$. Isolation between the modes may be improved (if inadequate) by insertion of resonant irises ${ }^{12}$ at $\mathrm{P} 1$ and $\mathrm{P} 2$, designed to be transparent for one mode and reflective for the other.

The dimensions were chosen to give unloaded cavity resonances at $6.40 \mathrm{GHz}$ for $B_{1}$, and $5.70 \mathrm{GHz}$ for $B_{2}$. Choosing the cavity length $\left(a_{y}\right)$ to be a convenient $12 \mathrm{~cm}$ consistent with the requirements above specifies $a_{x}$ to be $2.89 \mathrm{~cm}$, and $a_{z}$ to be $3.49 \mathrm{~cm}$. These dimensions completely determine the size of the main cavity. The width of antechamber A1 and the height of antechamber A2 are chosen to give the antechambers a cross-section that is similar to that used for C-band microwave waveguide, to reduce attenuation and improve the Q. Unplated brass is used for the cavity walls because of its ease of construction, and is found to give Q's in the proper range.

\section{B. The shielded loop resonator}

For delivery of the third magnetic field mode, rf techniques are used to setup the desired field at $727 \mathrm{MHz}$. The design is based on a shielded loop resonator developed for 


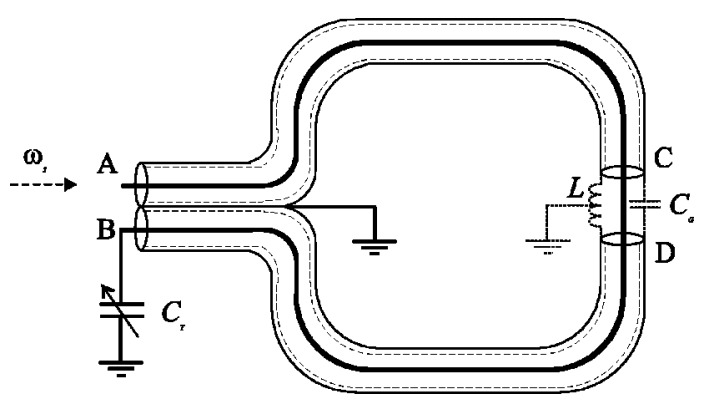

FIG. 3. The shielded loop resonator. The impedance at the gap in the shield between points $\mathrm{C}$ and $\mathrm{D}$ is the gap capacitance $\left(C_{G}\right)$ in parallel with the inductance around the outer part of the shield $(L)$, shown schematically by the dashed components. Changing the capacitance, $C_{T}$, tunes the resonant frequency of the loop.

magnetic resonance imaging. ${ }^{13,14}$ A schematic of the shielded loop is shown in Fig. 3. The device consists of a length of semirigid coaxial cable that is bent into a closed loop (the exact shape is not critical) with the shield (outer conductor of the coax) shorted together at the feedpoints. The shield directly opposite the feedpoint is completely removed from the cable, creating a gap at that position. There are many methods that can be used to drive the loop at the feedpoints. One method that has been found to be particularly simple is using an unbalanced feed to drive the loop at one of the feedpoints (A), and coupling the other feedpoint (B) to ground with a tuning capacitor, $C_{T}$.

Operation of the resonator is similar to that of a simple magnetic dipole loop antenna, with some important distinctions. The presence of the shield around the central conductor prevents significant leakage of electric fields, except in the vicinity of the break in the shield between C and D. Instead, a strong resonant near-field magnetic standing wave is created. The absence of any significant electric fields out- side of the loop makes its resonance characteristics largely impervious to dielectric loading and reduces capacitively mediated losses.

For rf signals, the skin depth of the conductive material of the shield is much less than the shield's thickness. This has the effect of electrically isolating the inner and outer sides of the shield everywhere except at the gap and feedpoints. ${ }^{14}$ Current in the central conductor induces an equal current in the opposite direction on the inner side of the shield. Current on the inside of the shield divides at $\mathrm{C}$ or $\mathrm{D}$ into current in the opposite direction on the outside of the shield, and a capacitive charge buildup across the gap. The net current in the structure can then be thought to be solely on the outside of the shield. It is this current that sets up a resonant magnetic field in the near-field of the loop, implying that the outer part of the shield should be thought of as an inductor. Once the standing wave has been formed, all current in the loop must have the same phase, and has mirror symmetry between the top (A-C side) and bottom (B-D side). Therefore, the magnetic field standing wave outside the loop generated by this current also has a definite phase, independent of position. As long as the loop is operated in a mode where the resonant wavelength is at least twice the loop circumference, the magnetic fields generated at a given point outside the structure by infinitesimal current segments around the loop add constructively.

The dashed components in Fig. 3 are used for modeling purposes to show that the total impedance across the gap in the shield (between C and D) is comprised of the gap's capacitance $\left(C_{G}\right)$ in parallel with the inductance of the outer part of the shield $(L)$. A semidistributed circuit analysis that makes no assumptions about the size of the loop compared to the input wavelength finds the impedance of the loop between point $\mathrm{A}$ and ground to $\mathrm{be}^{14}$

$$
Z=i Z_{0} \frac{\sin (\omega \tau)\left[\omega L+2 Z_{0}^{2} \omega C_{T}\left(1-\omega^{2} L C_{G}\right)\right]+\cos (\omega \tau)\left[Z_{0} \omega^{2} L C_{T}-2 Z_{0}\left(1-\omega^{2} L C_{G}\right)\right]+Z_{0} \omega^{2} L C_{T}}{\cos (\omega \tau)\left[\omega L+2 Z_{0}^{2} \omega C_{T}\left(1-\omega^{2} L C_{G}\right)\right]-\sin (\omega \tau)\left[Z_{0} \omega^{2} L C_{T}-2 Z_{0}\left(1-\omega^{2} L C_{G}\right)\right]-\omega L}
$$

where $Z_{0}$ is the impedance of the coaxial cable, $\tau$ is the travel time of an electronic signal around the loop, and $\omega$ is the driving frequency. At resonance, a maximum in the stored energy within the cable occurs at the poles of the loop's impedance, or equivalently when the reactance of the loop goes from inductive (positive) to capacitive (negative). Thus, those frequencies that make the denominator vanish in Eq. (3) are the resonant modes of the loop, $\omega_{0}$ :

$$
\begin{gathered}
\cos \left(\omega_{0} \tau\right)\left[\omega_{0} L+2 Z_{0}^{2} \omega_{0} C_{T}\left(1-\omega_{0}^{2} L C_{G}\right)\right]-\sin \left(\omega_{0} \tau\right) \\
\times\left[Z_{0} \omega_{0}^{2} L C_{T}-2 Z_{0}\left(1-\omega_{0}^{2} L C_{G}\right)\right]=\omega_{0} L
\end{gathered}
$$

There are multiple solutions to this equation, but the loop has the highest $\mathrm{Q}$ factor when it is operated in the fundamental mode, for which $\omega \tau \sim \pi$. That is, the resonant wavelength inside the coaxial cable is roughly twice the circumference of the loop. By changing the feed capacitance, $C_{T}$, the resonant frequency is tuned, essentially allowing the loop characteristics (cable impedance, velocity, and loop circumference) to be set by other design considerations.

A loop with a circumference of $12.8 \mathrm{~cm}$ is used in the trimodal resonator, made from a semirigid, $2.2 \mathrm{~mm}$ diameter, $50 \Omega$ cable (Storm Products, 421-193) with a velocity of propagation specified to be $0.78 c(\tau=0.55 \mathrm{~ns})$. It is chosen for its superior temperature-dependent phase stability and small cross-section. The conductors are copper, separated by a teflon dielectric. The tuning capacitor is a nonmagnetic, high-Q device designed for NMR applications (Johanson, 56401). 


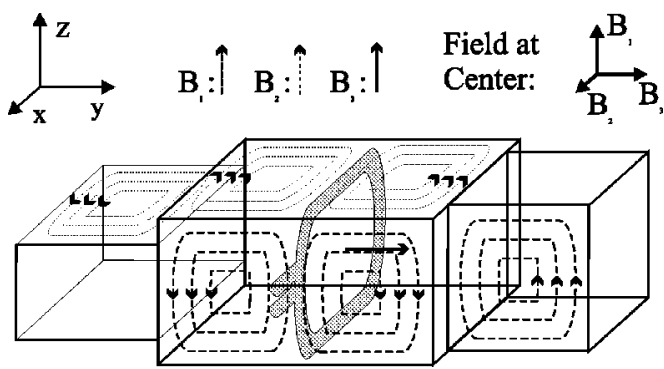

FIG. 4. A schematic of the trimodal resonator, showing the overall magnetic field configuration.

\section{The trimodal resonator}

To complete the trimodal resonator, the B-field generated by the shielded loop $\left(B_{3}\right)$ must overlap and be perpendicular to both $B_{1}$ and $B_{2}$ within the interaction region at the center of the main cavity. Due to the skin effect discussed earlier, the magnetic field generated by a shielded loop placed around the outside of the bimodal resonator (oriented in the $x z$ plane, coaxial with the resonator) will induce eddy currents on the outer surface of the resonator having a direction exactly opposite that of the current in the outer part of the shielded loop. The effect will be almost a perfect cancellation of the magnetic field at the center of the main cavity. However, placing the shielded loop on the inside of the bimodal resonator as shown in Fig. 4 has been found to generate a magnetic field at the interaction region that is nearly indistinguishable from the field generated by the loop alone.

Figure 4 indicates the magnetic field configuration for all three modes operating simultaneously in the resonator. Note that though the three fields are not perpendicular throughout the whole cavity, they are perpendicular (with minimal gradients) in the central interaction volume of a few $\mathrm{mm}^{3}$ occupied by the rubidium atoms of interest.

The presence of the metal and dielectric of the coaxial cable alter the resonance characteristics of the bimodal resonator only slightly. As long as a small cross-section loop is placed adjacent to the walls of the cavity $\left(E_{1}\right.$ approaches zero at the cavity walls that lie in the $y z$ plane, and $E_{2}$ approaches zero at the cavity walls that lie in the $x y$ plane of Fig. 4) and at an E-field node, the detuning effects of the loop's dielectric and the exclusion of electric flux are minimal. The exclusion of magnetic flux by the loop could in principle significantly tune the resonant frequencies of the cavity higher since the magnetic fields are not small in the central nodal plane at the cavity walls. However, the detuning effect of the loop on the bimodal cavity modes was measured to be very small ( $\sim 5 \mathrm{MHz}$ ). Thus, a loop with a semirectangular shape is used to minimize its distance from the cavity walls. The two ends of the loop are brought out of the bimodal cavity through a hole in one of the side walls, and wired as discussed above. The shield needs to be electrically isolated from the walls of the cavity, and is grounded at the feedpoints.

\section{TESTING THE CAVITY}

The first tests performed on the trimodal resonator demonstrated the modes' tunability. A small pick-up loop

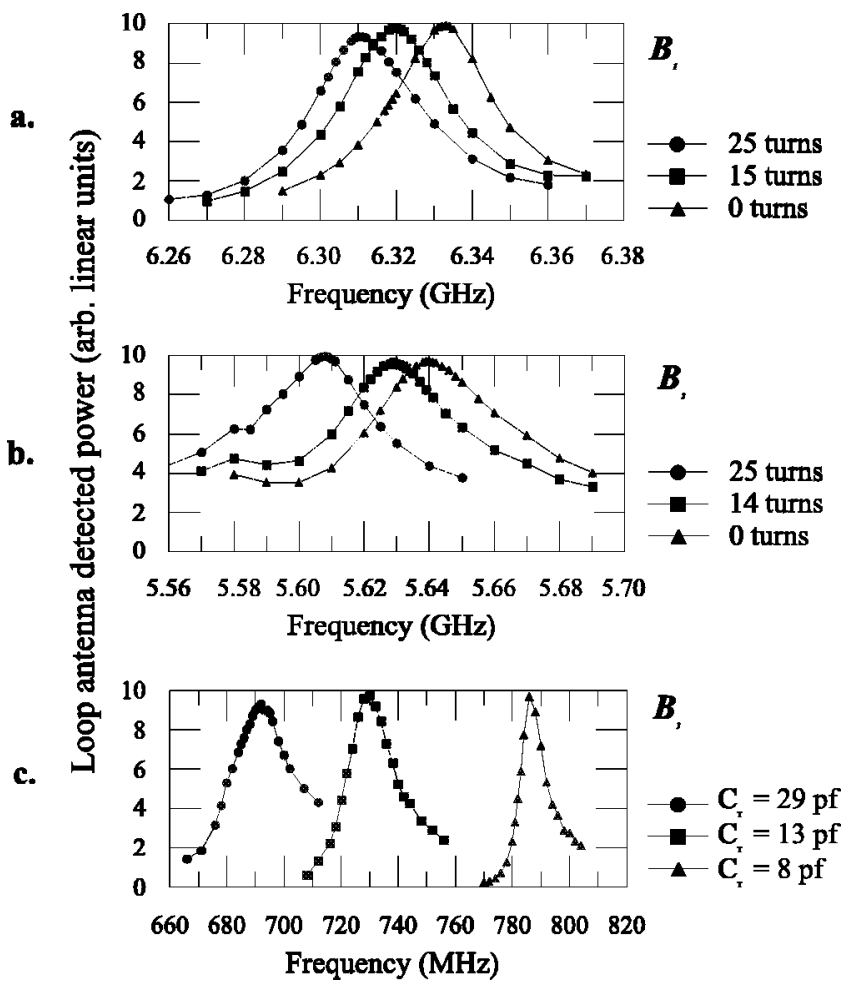

FIG. 5. Resonance plots and tuning for each mode. (a) is data for mode one, (b) is data for mode two, and (c) is data for mode three. The number of turns in (a) and (b) corresponds to the depth of the (nylon) screws shown in Fig. 2.

(slightly larger than the interaction region) in series with a zero-bias Schottky diode (M-Pulse, mp 2072) inserted into the center of the cavity served as an antenna-fed square-law detector, giving a voltage directly proportional to the stored power in the field. Initially, we verified that each mode had a magnetic field with the proper polarization by placing the plane of the loop antenna normal to the B field for that mode. The antenna was then oriented with components of all three B fields perpendicular to its plane. Measurements were made of the resonant frequency and relative power in the three fields. With the tuning screws removed from the bimodal cavity, the resonances of these two modes were measured to be 5.64 and $6.33 \mathrm{GHz}$. These resonant frequencies are slightly lower than those predicted by Eq. (2) because of the perturbations to the cavity. With the tuning capacitor on the shielded loop set to a minimum (7 pf), the resonant frequency was found to be about $800 \mathrm{MHz}$. Tuning data for each mode driven with the other two off is shown in Figs. 5(a)-5(c). Nylon screws were used for the data in Figs. 5(a) and 5(b), indicating a tuning range for each mode of about $35 \mathrm{MHz}$. Alumina screws were also used to tune the resonator, and were found to provide a tuning range of about 100 $\mathrm{MHz}$ for each mode.

In addition, reflection measurements were made for a range of data points around the resonant frequency, as shown in Figs. 6(a)-6(c). The reflected power was measured by placing a directional coupler at each input of the trimodal resonator, such that reflections from the cavity were sent to a diode (Hewlett Packard crystal detector 432A) for rectification, giving a voltage proportional to reflected power. These 


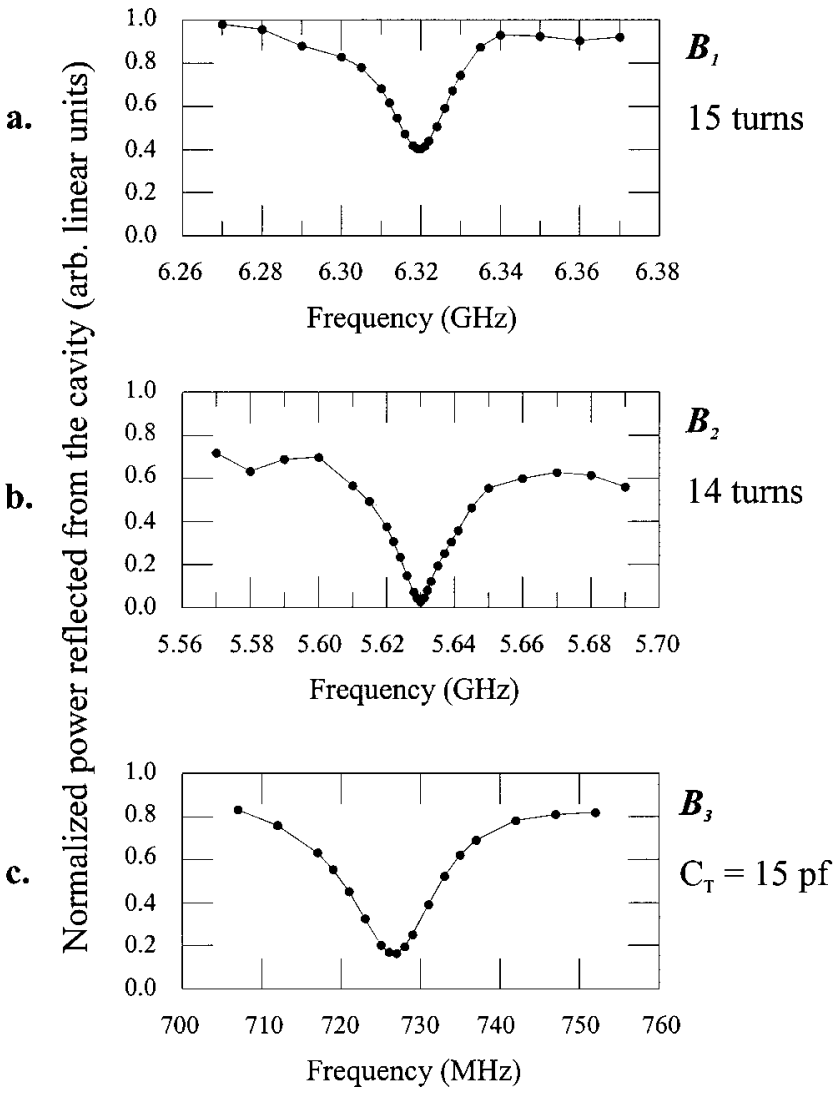

FIG. 6. Measurements of the reflected power from the input port for each mode driven near resonance.

plots show that the reflected power passed through a minimum at resonance, indicating energy was efficiently transferred from the transmission line to the standing wave fields.

Figure 7 shows a least-squares regression analysis for the resonant frequency of $B_{3}$ versus tuning capacitance, $C_{T}$. Fit parameters from the model give values for $C_{G}(7.0 \mathrm{pf})$ and $L(17 \mathrm{nH})$, both near approximate expected values, showing that Eq. (4) provides a sufficient description of the resonance condition for the loop.

These data also give a measurement of the $\mathrm{Q}$ for each mode, shown in Table $\mathrm{I}$, where the $\mathrm{Q}$ is defined as $\omega_{0} / \Delta \omega$ and $\Delta \omega$ is the full-width at half-maximum (FWHM) of the stored energy versus frequency line shape. Typical Q's for $B_{1}$ and $B_{2}$ are about 200 and 150, respectively. Typical Q's

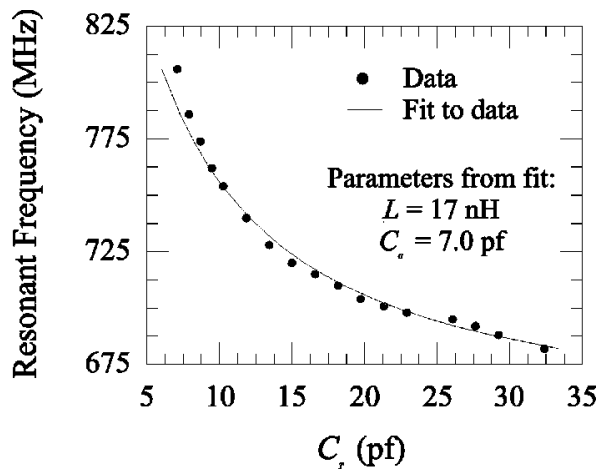

FIG. 7. Tuning data for the resonant frequency of $B_{3}$ fit to Eq. (4). $C_{T}$ is a tuning capacitor placed at a feedpoint for the shielded loop. The values of $C_{G}$ and $L$ are obtained from the fit.
TABLE I. Summary of data from the trimodal resonator.

\begin{tabular}{ccccc}
\hline \hline$B$ field & Polarization & Resonant frequency & Tuning range & $Q_{\text {unloaded }}$ \\
\hline$B_{1}$ & $\hat{z}$ & $6.3 \mathrm{GHz}$ & $100 \mathrm{MHz}$ & 200 \\
$B_{2}$ & $\hat{x}$ & $5.6 \mathrm{GHz}$ & $100 \mathrm{MHz}$ & 150 \\
$B_{3}$ & $\hat{y}$ & $0.7 \mathrm{GHz}$ & $125 \mathrm{MHz}$ & $\sim 45$ \\
\hline \hline
\end{tabular}

for $B_{3}$ vary between 25 and 65 , depending on the value of $C_{T}$. Preliminary measurements were also taken with the cavity loaded with a $2.8 \times 3.4 \times 1 \mathrm{~cm}$ rubidium-filled quartz cell. The loaded Q's of $B_{1}$ and $B_{2}$ were still in the range of 100-200, indicating that the cell has little effect on the field geometry and node placement inside the resonator. Loading had no measurable effect on the resonant lineshape of $B_{3}$, consistent with the supposition that the shielded loop produces very little $\mathrm{E}$ field.

In order to obtain a measurement of the isolation between each of the modes, the rectified voltages at the inputs to two of the ports were monitored while the third mode was driven at resonance. The isolation between any two modes was measured to be better than $22 \mathrm{~dB}$, a result of the geometric perpendicularity between the modes. Also, with the shielded loop driven on resonance no field was measured in $B_{1}$ and $B_{2}$ (the detector noise places the lower limit on the isolation measurement at $40 \mathrm{~dB}$ ) due to the fact that this frequency is well below cutoff for the bimodal resonator.

Finally, measurements were made of the phase control within the structure. A measurement of the overall phase $\Phi$, defined in Eq. (1), was obtained by placing the antenna-fed rectifying diode loop into the cavity. The three frequencies were mixed by the diode, producing sum and difference frequencies (and higher harmonics), giving a de level that has a dominant term proportional to $\sin (\Phi)$. This type of phase measurement is only possible when $\omega_{1}+\omega_{2}=\omega_{3}$. For the following phase measurements, the three modes were tuned to be resonant at $6.33 \mathrm{GHz}$ for $B_{1}, 5.61 \mathrm{GHz}$ for $B_{2}$, and $0.72 \mathrm{GHz}$ for $B_{3}$, and were driven at these frequencies. By varying the input phase of the field fed into the cavity for any one of the three signals, the dc level at the output of the diode should vary accordingly. For the data shown in Fig. 8, $\varphi_{1}$ was varied by changing the path length of the input transmission line, using an air-filled $50 \Omega$ variable delay line. The detected power is plotted versus the delay length of the transmission line, in units of the known wavelength for that mode $\left(\lambda_{0}=47.3 \mathrm{~mm}\right)$. Since Fig. 8 displays results from a loop probe, it is a measurement of the overall B-field phase in the

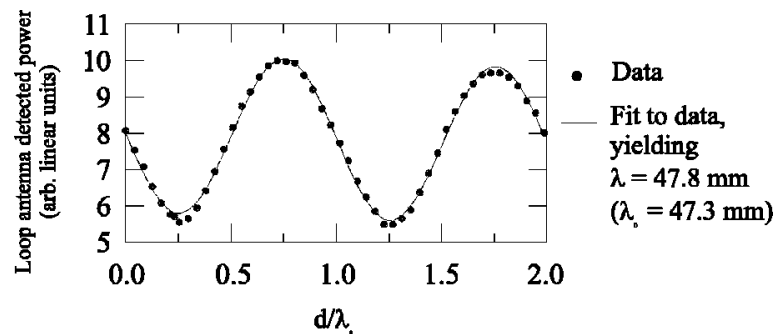

FIG. 8. B-field phase measurements in the trimodal resonator. The phase of $B_{1}$ in the cavity is varied by changing the length of the input transmission line, $d$. $B_{1}$ is driven at $6.33 \mathrm{GHz}, B_{2}$ is driven at $5.61 \mathrm{GHz}$, and $B_{3}$ is driven at the difference frequency, $0.72 \mathrm{GHz}$. 
resonator. The fit wavelength is found to be consistent with the known wavelength at a level of $1 \%$, indicating that precise relative phase control of the modes inside the resonator is obtainable.

\section{APPLICATION TO T-INVARIANCE EXPERIMENT}

In order for the trimodal resonator to be used for the time-reversal experiment in rubidium, several modifications are necessary. Signal from rubidium indicating population in one of the three sublevels of the ground state is obtained through excitation by two weak frequency-stabilized probe lasers followed by fluorescence detection. To collect the fluorescent light, part of one side of the bimodal resonator was replaced by a thin copper mesh, characterized by a spacing of 70 lines per inch and an overall transparency of $\sim 87 \%$. This mesh was found to have little effect on the resonance properties of the cavity.

In addition, to prepare the rubidium in the proper initial state for the experiment, an optical pumping laser is used, requiring another hole in the cavity. In all, two $3 \mathrm{~mm}$ diameter holes are needed for the lasers, one $5 \mathrm{~mm}$ hole is required for the stem of the quartz cell used to hold the rubidium, one $25 \mathrm{~mm}$ hole covered by the copper mesh is used for fluorescent light collection, one $2 \mathrm{~mm}$ hole is required for the rectified wire loop probe, and a hole is added for the leads of the shielded loop. With these perturbations in place, the cavity is tuned to the proper resonant frequencies, and heated to approximately $100{ }^{\circ} \mathrm{C}$. A dc magnetic field of 806 Gauss is applied in the $z$ direction, and fluorescence is monitored, initially with the three ac coupling magnetic fields off. With the $\sigma^{-}$optical pumping laser on, the rubidium is preferentially placed in the low angular momentum state $(\mid 2$, $-2\rangle$, see Fig. 1). Fluorescence is minimized while this state is populated, and is roughly proportional to the population in all of the other substates of the ground state. The application of either of the two fields that couple population out of $\mid 2$, $-2\rangle$ (the two lower frequency fields), should thus increase the amount of fluorescence. This is in fact exactly what was observed during initial data collection, indicating that the resonator produces appreciable magnetic field standing wave modes $B_{2}$ and $B_{3}$ with the stated polarizations.

In summary, we have demonstrated a tunable trimodal resonator for three perpendicular magnetic fields. Each mode is independently tunable, and the Q's are approximately 200, 150 , and 45 for $B_{1}, B_{2}$, and $B_{3}$, respectively. The resonator has been observed to couple magnetic dipole transitions in rubidium, and will soon be used to coherently drive a threelevel system to test time-reversal symmetry in an atomic system.

\section{ACKNOWLEDGMENTS}

The authors wish to thank Richard Sands and Richard Dunham for helpful discussions about ESR. We are also grateful to Pete Peterson for his work on the prototype for the degenerate bimodal cavity. This work was supported by NSF Grant No. PHY-9417854 and the University of Michigan.

${ }^{1}$ S. J. Buckle, S. M. Barnett, P. L. Knight, M. A. Lauder, and D. T. Pegg, Opt. Acta 33, 1129 (1986).

${ }^{2}$ R. S. Conti, "Search for T-odd, P-even Interactions with a Three-Level Atomic Clock," Time Reversal-The Arthur Rich Memorial Symposium, edited by M. Skalsey, P. H. Bucksbaum, R. S. Conti, and D. W. Gidley (AIP, New York, 1993).

${ }^{3}$ K. Mobius, M. Plato, and W. Lubitz, Phys. Rep. 87, 171 (1982).

${ }^{4}$ T. C. Christidis, H. S. Mchaourab, and J. S. Hyde, J. Chem. Phys. 104, 9644 (1996).

${ }^{5} \mathrm{H}$. Thomann and M. Bernardo, Chem. Phys. Lett. 169, 5 (1990).

${ }^{6}$ D. A. Hall, D. C. Maus, G. J. Gerfen, S. J. Inati, L. R. Becerra, F. W. Dahlquist, and R. G. Griffin, Science 276, 930 (1997).

${ }^{7}$ A. Ramamoorthy (personal communication).

${ }^{8}$ J. S. Hyde, J. C. W. Chien, and J. H. Freed, J. Chem. Phys. 48, 4211 (1968).

${ }^{9}$ M. Huisjen and J. S. Hyde, Rev. Sci. Instrum. 45, 669 (1974).

${ }^{10}$ N. Marcuvitz, Waveguide Handbook (McGraw-Hill, New York, 1951), p. 307.

${ }^{11}$ J. D. Jackson, Classical Electrodynamics (Wiley, New York, 1975), p. 349.

${ }^{12}$ W. C. Caldwell, "Bandpass TR Tubes," Microwave Duplexers, edited by L. D. Smullin and C. G. Montgomery (Wiley, New York, 1948).

${ }^{13}$ M. D. Harpen, Magn. Reson. Med. 32, 785 (1994).

${ }^{14}$ A. Stensgaard, J. Magn. Reson., Ser. A 122, 120 (1996). 